\title{
La investigación como herramienta de formación continuada para la docencia y viceversa*
}

\author{
Research as a Tool of Continuing Training for Teaching and vice versa \\ A pesquisa como ferramenta de formação continuada para docência e vice-versa
}

Fecha de recibido: 10 de noviembre de 2019 | Fecha de aceptado: 15 de mayo de 2020 | Fecha de publicación: 20 de agosto de 2020

\author{
Elisabete Andrade ${ }^{\mathrm{a}}$ \\ Universidade Regional do Noroeste do Estado do Rio Grande do Sul (UNIJUÍ) / RS, Brasil \\ ORCID: orcid.org/0000-0002-3517-8162
}

\author{
Jaime Moreles Vázquez \\ Universidad de Colima, México \\ ORCID: orcid.org/0000-0002-1830-6177
}

* Artículo de revisión de la literatura dirigida a la reflexión sobre el tema estudiado.

aAutora de correspondencia: andradeelisabete15@gmail.com

doi: https://doi.org/10.11144/Javeriana.uh89.ihfc

Cómo citar este artículo: Andrade, E. y Morales Vásquez, J. (2020). La investigación como herramienta de formación continuada para la docencia y viceversa. Universitas Humanística, 89. https://doi.org/10.11144/Javeriana.uh89.ihfc 


\title{
Resumen
}

Pensar en la docencia como posibilidad de investigación y la investigación como proceso formativo, ambos articulados con lo que se ha denominado educación continua para docentes, es el objetivo del texto que aquí se presenta. Tomamos la docencia, la formación continuada y la investigación como ejes problematizadores, argumentando la necesidad de tener la docencia como fundamento de la investigación y esta última como fundamento de la primera. La formación continua de los docentes es vista como base articuladora de los procesos viceversa que abordamos en el desarrollo de la argumentación. Partimos de la revisión bibliográfica estudiando las concepciones de investigación y su articulación o desarticulación con la docencia. Revisamos los enfoques de la formación continua del profesorado, comprendiendo los conocimientos teóricos y conceptuales sobre estos movimientos de formación. Interpretamos el "objeto" en estudio desde la perspectiva analítica, utilizando el mito de Sísifo como metáfora provocadora del pensamiento.

Palabras clave: investigación; enseñando; educación continua

\begin{abstract}
Thinking about the teaching practice as a research possibility for research and research as an educational process, both of them articulated with something that is called continuing training for teachers, is the aim to accomplish in this paper. We take the teaching, the continuing training and the research as the central issues and discuss the need to assume the teaching as a fundamental for research and, in turn, the research as a fundamental for the former. Continuing training of teachers is deemed as the basis articulating the double-way processes we use to development our arguments. We start by a bibliographic review and study the conceptions of research and whether they are or not articulated with the teaching practice. We reviewed the approaches of continuing training by the teachers in order to understand the theoretical and conceptual knowledge
\end{abstract}


regarding these educational movements. We interpret the "object" under study from an analytical perspective, using the myth of Sisyphus as a metaphor eliciting the thinking.

Keywords: research, teaching, continuing education

\section{Resumo}

Pensar a docência como possibilidade de pesquisa e a pesquisa como processo formativo, os dois articulados com o que se nomeio de educação continua para docentes, é o objetivo do texto aqui apresentado. Tomamos a docência, a formação continuada e a pesquisa como eixos problematizadores, argumentando a necessidade de ter a docência como fundamento da pesquisa e esta última como fundamento da primeira. A formação continua dos docentes é vista como base articuladora dos processos vice-versa que abordamos no desenvolvimento da argumentação. Partimos da revisão bibliográfica estudando as concepções de pesquisa e sua articulação ou desarticulação com a docência. Revisamos as abordagens da formação continua dos professores, a compreender os conhecimentos teóricos e conceptuais sobre esses movimentos de formação. Interpretamos o "objeto" de estudo desde a perspectiva analítica, utilizando o mito de Sísifo como metáfora instigadora do pensamento.

Palavras-chave: pesquisa; ensinando; formação continuada

\section{Retorno a Sísifo}

Rodó la piedra y otra vez como antes la empujaré, la empujaré cuestarriba para verla rodar de пиеvo 
Comienza la batalla que he librado mil veces contra la piedra y Sísifo y mí mismo

Piedra que nunca te detendrás en la cima: te doy las gracias por rodar cuesta abajo. Sin este drama inútil sería inútil la vida

Pacheco, Tarde o temprano (Poemas 1958-2009)

\section{Distinciones conceptuales y teóricas}

En el presente trabajo discutimos una de las dimensiones de la compleja relación entre la investigación y la docencia en el campo de la educación, que consiste en la simbiosis entre tales actividades en lo relativo a la formación continua y en la significación de ambas prácticas. Para ese propósito proponemos una estrategia de argumentación que hemos denominado viceversa, pues la manera como abordamos el asunto en la primera parte va en un sentido, docenciainvestigación, para luego retornar sobre el mismo eje de discusión en la segunda parte, investigación-docencia.

Con la estrategia de argumentación viceversa pretendemos demostrar el movimiento dialéctico que atraviesa los procesos de formación de profesores en lo que se refiere al entendimiento de la investigación como constitutiva de la docencia y a la docencia como espacio-tiempo en el que se producen conocimientos y, por esa misma razón, posee potencial para ser fundamento de la investigación. Hablamos de un movimiento dialéctico en el sentido de que estos procesos presentan tensiones respecto a quién puede ser denominado investigador y quién puede ser reconocido como docente, y viceversa. Nuestra comprensión de tales tensiones va hacia las posibilidades de encuentro, proponiendo la formación continua como eje articulador. Pretendemos que esas argumentaciones sean dos sentidos que se encuentran en su acepción de significación de nuestras actividades y nuestra identidad como docentes e investigadores, como docentes-investigadores.

Por tanto, en la primera parte planteamos que la investigación representa una herramienta de formación continua para la docencia. Pensamos la investigación como un modo de conexión entre los procesos de teorización y de desenvolvimiento de las prácticas pedagógicas. En este 
sentido es que empleamos el término simbiosis. Según el diccionario Aurélio (Ferreira, 2010, p. 700), la palabra simbiosis puede ser traducida como una "asociación recíproca de dos o más organismos diferentes que les permite vivir beneficiándose uno del otro". Aludimos a la definición de este término para demostrar que, aunque la investigación posee modos de organización y estructuración propios, puede contribuir a la reflexión sobre los procesos mediante los cuales se desarrollan las prácticas pedagógicas en las instituciones educativas. Desde la investigación es posible concretar otras modalidades del ejercicio de la docencia, pues esta posibilita la indagación, la problematización, y el ir y venir argumentativo y de pensamientos que conducen a las reflexiones sobre/en/con la práctica pedagógica (Schön, 1992).

Volviendo sobre ese eje, en la segunda parte argüimos que para la formación continua de los investigadores para la investigación uno de los mecanismos más fecundos lo representan precisamente las actividades docentes. Entendemos la formación continua como un espaciotiempo propicio para el cuestionamiento y la reflexión de las actividades que cotidianamente se realizan en las instituciones educativas, dando lugar así a la investigación y la producción de conocimientos.

Concebir la formación continua a partir de referentes de la investigación ha sido poco frecuente en las discusiones al respecto, al contrario, este espacio de formación se ha asumido como algo distanciado de esta y, en consecuencia, de la reflexión como un elemento primordial. Para comprender la necesidad de la interrelación entre la investigación, la formación continua y la docencia proponemos la presente discusión. Una representación global de esta articulación se puede observar en la figura 1. 


\section{Figura 1. Representación global}

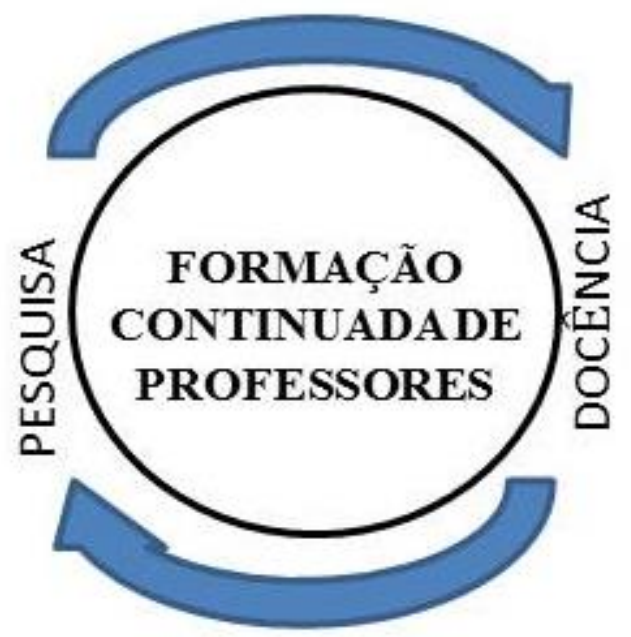

Fuente: elaboración propia

\section{Enfoque del concepto de campo}

Para la problematización propuesta aquí, trabajamos con el concepto de campo. Según Bourdieu (2004, pp. 22-23), cada campo "es un campo de fuerzas y un campo de luchas para preservar o transformar este campo de fuerzas". Debido a que entendemos la educación como un campo de luchas en el cual las disputas siempre están en contexto, tensionando los procesos formativos, es que tomamos concepto y a lo largo del texto operamos con la noción de campo de la educación. Los campos están formados por agentes, que pueden ser individuos o instituciones, los cuales crean espacios y los hacen existir a través de las relaciones que establecen allí. Uno de los principios de los campos, ya que determina lo que los agentes pueden y no pueden hacer, es la "estructura de relaciones objetivas entre diferentes agentes" (Bourdieu, 2004, p. 23). Es el lugar que ocupan los agentes en esta estructura lo que indica sus posiciones (Pereira, 2015). Por lo tanto, en el análisis formativo evidenciado aquí, ambos agentes-instituciones están en juego, porque cuando se trata del proceso de capacitación docente existe una inmanencia de lazos institucionales y, aun, agentes docentes en el contexto del trabajo pedagógico.

El objeto de estudio presentado aquí, la educación continua de los maestros, es en sí mismo un campo de luchas y disputas, por lo tanto, el concepto de campo de Bourdieu se vuelve apropiado. 
Es en esta perspectiva que dicha noción aparece en diferentes momentos del texto, e incluso, si bien indirectamente, termina componiendo el cuerpo teórico y analítico del objeto investigado.

\section{La formación continua}

Es importante agregar que concebimos la formación continua de profesores como una modalidad formativa que acontece después de la formación inicial. Es un momento importante de este proceso considerando que puede contribuir al desarrollo profesional, pues se refiere a temas y asuntos que afectan tanto a las prácticas pedagógicas cotidianas, como a las instituciones educativas en general, en todos sus niveles y modalidades.

Según Estrela y Estrela (2006), la formación continua comprende un conjunto de actividades encuadradas institucionalmente que, después de la formación inicial, pretenden el perfeccionamiento profesional y personal del profesor para conseguir un adecuado ejercicio de su función, siempre en beneficio de los alumnos y la escuela.

Desde esta perspectiva, la formación continua es comprendida como concepto normativo, vinculado a una necesidad de coherencia, lo que significa que es el comienzo de un proceso, no un fin en sí mismo. En ese sentido, la sola institucionalización de los principios no garantiza la eficacia de las actividades de formación, pues también es necesaria tanto la colaboración entre los ámbitos normativos e institucionales, como la implicación profesional de quienes trabajan en las instituciones educativas.

Cuando hablamos de investigadores y docentes nos referimos a los profesionales formados en los programas educativos de licenciatura y posgrado que desarrollan tanto actividades de investigación y enseñanza, como labores de gestión. En nuestra argumentación no diferenciamos al investigador del docente debido a nuestra comprensión de la investigación como fundamento de los procesos de enseñanza y de aprendizaje que acontecen en los procesos educativos en los niveles básico y superior. Sabemos que esta comprensión es un poco distinta a como hasta ahora se ha interpretado la cuestión, especialmente en el contexto de la educación básica, ya que de manera general se ha asumido que la investigación ocurre en el contexto de la educación superior, constituyéndose como uno de los objetivos de los programas de maestría y doctorado.

Aunque enseñanza e investigación no necesariamente puedan ser vistos como sinónimos, nuestros planteamientos van en ese sentido. Sabemos que para que esto se concrete es necesario 
que los docentes de educación básica puedan desarrollarse profesionalmente como investigadores de su propia práctica pedagógica, aunque la inversión de recursos para este propósito ha sido destinada principalmente a la educación superior y a los programas de posgrado.

\section{La investigación}

Analizando el sentido etimológico de la palabra "investigación” podemos encontrar que se deriva del término latino perquirere, que significa "buscar con perseverancia". También se traduce como un conjunto de acciones encaminadas a descubrir nuevos conocimientos en un área en particular. Por ejemplo, en el mundo académico la investigación es uno de los pilares de la actividad universitaria, donde los investigadores tienen como objetivo producir conocimiento desde y para alguna disciplina, contribuyendo así al avance de la ciencia y, en ocasiones, al desarrollo sociali ${ }^{i}$ En lo que concierne a pesquisar, comparte su origen en el latín perquirere, que significa "indagar, preguntar", y se compone de per-, “completamente”, y quaerere, "preguntar". ii Ambos significados, investigación e investigar, muestran aspectos relevantes que se complementan entre sí y contribuyen a la reflexión que estamos desarrollando para discutir la investigación como herramienta de formación continua del ejercicio de la docencia, y viceversa. Buscar con perseverancia nos hace pensar en la persistencia, la cual es justo lo que sugiere el mito de Sísifo que presentamos como epígrafe para proponer que la formación continua es esencial para las actividades cotidianas de las instituciones educativas, teniendo como marco de referencia la simbiosis entre la docencia y la investigación.

De esta forma, incorporando a nuestra comprensión el constante y persistente movimiento de "rodar la piedra", y a la vez pensar, indagar, preguntar, es decir, investigar tanto la propia actividad docente como los límites y las posibilidades que se nos presentan en el espacio-tiempo en el que esta acontece, consiste en adquirir una mirada inquisitiva y una escucha atenta a los detalles, lo que podemos denominar conceptualmente la estética de lo cotidiano. Sobre esta cuestión abundamos en la siguientes líneas.

\section{Macroestética y microestética: la significación de lo cotidiano}

La estética de lo cotidiano comprende los objetos o las actividades presentes en la vida cotidiana como poseedores de un valor estético para la cultura correspondiente, principalmente la 
subjetividad de los sujetos que la constituyen, y cuya estética se organiza a partir de múltiples facetas de su proceso de vida y de transformación (Richter, 2003, p. 21).

Pereira (1996, p. 85) desarrolla lo que denomina un "intento de resignificación” de la estética que, según Richter (2003, p. 21), "puede ser muy útil para el estudio de la estética de lo cotidiano". El autor trata de establecer una "diferencia dentro de la estética" mediante las designaciones de "macroestética y microestética". La primera alude a una estética con "E" mayúscula que nace en el siglo XVIII como un campo epistemológico independiente, como una disciplina; y la microestética "se refiere a la forma en que cada individuo se organiza en cuanto a su subjetividad; es del orden de procesualidad, de los campos de fuerzas vivas de la exterioridad que atraviesa un sujeto-en-práctica” (Richter, 2003). Según el autor, la macroestética es producto de una subjetividad que se quiere instituir como modelo homogeneizante, por ejemplo, en los conceptos de belleza, de creatividad, de lo que se considera investigación o docencia, mientras que la microestética se refiere al proceso de producción de subjetividades. De esta manera, la estética tiene que ver con la manera por la cual el mundo tiene sentido para nosotros, según la forma como nos afecta y como la afectamos nosotros (Pereira, 1996, p. 127).

Cuando se trata de microestética, Pereira (1996) y Richter (2003) consideran las diferentes formas mediante las que los sujetos habitan el mundo; en el caso de la presente argumentación, se trata de las formas como los maestros e investigadores se refieren y se relacionan con los procesos de formación continua y de desarrollo profesional a lo largo de su carrera. "Rodó la piedra y otra vez como antes la empujaré, la empujaré cuestarriba para verla rodar de nuevo [...]". ¿Qué es el trabajo cotidiano y la vida misma si no la constante rodada de las piedras? La simbiosis entre la investigación, la formación continua y la docencia sugiere la persistencia de empujar las piedras y verlas rodar tantas veces como sea necesario.

En este contexto está en juego la microestética (Pereira, 1996) con respecto a las formas en que subjetivamente damos cuenta de lo cotidiano del trabajo y de la vida diaria. La concebimos como potenciadora de las relaciones entre el mundo del trabajo y el de la vida, entre la investigación y la docencia, tanto en la educación básica como en la educación superior. Teniendo en cuenta las subjetividades, se entienden las diferencias que existen entre cada una las instancias que organizan la vida y el trabajo. La idea central consistiría en encontrar en la diferencia un espacio de interlocución y complementariedad, hallar un puente entra la macroestética y la microestética. 
Los referentes desde los que partimos son desarrollados a lo largo de todo el texto. De esta manera mostramos desde dónde hablamos y también la estrategia viceversa que proponemos. Resaltamos que el camino se construye mientras lo andamos (Marques, 2011), por eso, en términos metodológicos, la propia construcción argumentativa demuestra el camino que nosotros recorrimos para la comprensión de nuestro objeto.

El mito de Sísifo que empleamos como epígrafe constituye parte del proceso metodológico cuando lo entendemos como metáfora impulsora y conductora del pensamiento. Partimos desde esta metáfora para posicionarnos frente a la problemática, y adentrarnos en los conceptos de investigación, docencia y formación continua y su articulación, ya que las "metáforas no crean sistemas de pensamiento. Están sueltas y libres como mariposas viajeras [...]. Pertenecen al mundo de la vida. Quienes crean sistemas de pensamiento y acción son los conceptos que en ellas se encuadran" (Marques, 2011, p. 55).

Entendemos que el proceso metodológico, además de las normas prescritas y respetadas académicamente, también implica un proceso de creación, de "experiencia imaginativa" (Marques, 2011, p. 55), que muestra los caminos por los cuales se generan las diversas formas de pensar los fenómenos investigados. La "experiencia imaginativa" la entrelazamos aquí a los preceptos teóricos que traducen los conceptos de los cuales hablamos, construyendo así un modo de pensar y argumentar.

Con nuestro análisis proponemos ampliar las posibilidades de diálogo y la comprensión de la simbiosis entre la investigación, la formación continua y la docencia, independientemente del nivel en el que se actúa profesionalmente como profesor. La articulación de estos tres ejes puede estructurar la formación de docentes a lo largo de toda su carrera, favoreciendo el desarrollo de la práctica pedagógica problematizadora como propuso Freire (1990).

\section{Viceversa}

Primera parte: investigación como herramienta de formación continua de los profesores o la significación de lo cotidiano 
Con base en los planteamientos que hasta aquí hemos esgrimido, ¿qué condiciones y posibilidades de la investigación la tornan una herramienta de formación continua para los profesores?, ¿por dónde comenzar?

Pensando en el concepto de microestética y su relación con las subjetividades, es evidente que la manera como se ha desarrollado la formación continua acaba por generalizar y hasta homogeneizar los diversos contextos educativos, y, de este modo, no deja espacio a las características institucionales, ni a las subjetividades y especificidades de cada espacio-tiempo. Reducir la formación continua apenas a eventos externos a las instituciones educativas en los que los docentes actúan y ejercen sus prácticas, sea en forma de conferencias, seminarios, talleres, congresos, etc., equivale a disminuir las posibilidades de discusión y problematización de la práctica pedagógica que se desarrolla dentro de las aulas de educación básica y superior.

Además de la participación y el compromiso con los eventos externos, es importante que exista un movimiento formativo que se origine desde las instituciones y las subjetividades de los docentes para cuestionar y problematizar su propia práctica pedagógica. Este movimiento de reflexión en/sobre/con la práctica pedagógica (Schön, 1992) es lo que llamamos incorporar la investigación como fundamento formativo a la formación continua de los profesores, convirtiéndose en uno de sus principios.

Otra cuestión relevante en cuanto a la incorporación de la investigación a la formación continua de los profesores se refiere a la comprensión de que los profesores construyen sobre la práctica pedagógica que desarrollan en el aula y sobre las lecturas que hacen de la vida cotidiana de las instituciones en las que trabajan. Aquí encontramos una pista importante relacionada con la mirada y escucha de los profesores en su contexto profesional, considerando que uno de los aspectos que origina y orienta la investigación es la curiosidad sistemática, argumentativa y problematizadora de lo cotidiano. La investigación como una indagación sistemática y autocrítica que se basa en la curiosidad y en el deseo de comprender, una curiosidad estable, no fugaz, sistemática en el sentido de respaldarse en una estrategia, es fundamental en la indagación como temple escéptico, fortalecido por principios críticos, una duda no solo respecto a las respuestas obtenidas y cómodas, sino también acerca de las hipótesis propias (Stenhouse, 2007, p. 28). Esta relación formativa va al encuentro de la superación de los fundamentos centrados únicamente en la racionalidad instrumental, pues abre las posibilidades a otras formas de pensar 
y, por consiguiente, a otras maneras de desarrollarse profesionalmente y de practicar la docencia. La articulación de la tríada investigación, formación continua y docencia se llevará a cabo a partir de la indagación curiosa, responsable y crítica de lo cotidiano. Para que esto ocurra, otro aspecto relevante es aprender a hacer preguntas. Estas surgen cuando existe un sustento teórico que da lugar al deseo de conocer más allá de lo que ya está establecido, más allá del límite de las prácticas y las comprensiones que hemos construido sobre estas. El conocimiento teóricometodológico de determinado "objeto" es lo que permite las condiciones para generar nuevas preguntas y, de este modo, avanzar en la comprensión de lo que se está estudiando. En este caso, podríamos conjeturar que si los docentes no poseen un cuerpo de conocimientos teóricos que sustenten su práctica, difícilmente podrán articularlo a la tríada que aquí proponemos. Tampoco los investigadores lo lograrían.

Coincidimos con Stenhouse (2007) cuando afirma que para que la investigación resulte útil a los profesores, es necesario que ellos comprueben las implicaciones teóricas de las que esta surge, pues gran parte de la investigación educativa, en razón de su fidelidad al paradigma psicoestatístico, posee generalizaciones que no podrían brindar orientación para la acción en contextos específicos. La primera cuestión es acerca de la implicación teórico-práctica, es decir, la conciencia del cuerpo teórico que es inseparable de la práctica pedagógica puesto que es su fundamento. La segunda cuestión es que los significados teórico-prácticos no se pueden generalizar, pues cada espacio-tiempo educativo produce su propia cultura; sin embargo, en la incorporación y fusión cultural de la tradición siempre hay un proceso de reconstrucción a partir del tiempo y el lugar vivido, el puente entre la macroestética y la microestética. Es en este sentido que los contextos culturales merecen ser considerados e interpretados a la luz de un cuerpo de conocimientos científicos que puede traducir sus especificidades y, a partir de ellas, actuar.

También es importante que señalemos que cuando hablamos de la necesidad de reflexión por parte de los profesores, que es un proceso inherente a la problematización de lo cotidiano mediante la investigación, aducimos que implica un profundo proceso de elaboración del pensamiento y de permanente preocupación por los eventos que emergen de las prácticas pedagógicas. Desde esta perspectiva, la reflexión implica estar analizando y autoanalizando constantemente los eventos, y generando consecuencias o repercusiones en la vida cotidiana. Por esa razón, ser un profesor o investigador reflexivo no es algo sencillo, al contrario, consiste en 
asumir una postura activa, dialógica y transformadora. No hay investigación sin el acto de reflexión, no hay reflexión sin investigación.

Retornamos al mito de Sísifo para reiterar la complejidad de la relación que proponemos con la tríada investigación, formación continua y docencia, en cuanto exige un movimiento constante y persistente de un ir y venir reflexivo, para, en este proceso, irse autoformando, autorreconociendo, autoafirmando como profesor-investigador, un sujeto capaz de analizar con cuidado e inquietarse con lo que piensa, dice y hace.

Como mencionamos, partimos de la premisa de que docencia e investigación están íntimamente relacionadas y por esa razón empleamos el término simbiosis, la cual se construye por "el arte de la reflexión en la acción” (Schön, 1992, p. 10) y por intangibles como el oficio, el compromiso, el entusiasmo y la pasión, que no siempre son inherentes a las tareas académicas, y que han sido sugeridos por autores como Day (2011), Elliot (2000) y Freire (1990). A nuestro juicio, lo que el encuentro de las dos actividades genera es la revitalización de esos intangibles y la significación de las prácticas, como proponemos en lo que sigue.

\section{Segunda parte: la docencia como herramienta de formación continuada para la investigación o la pérdida/recuperación de la pasión}

Según Day (2011), los intangibles más esenciales en las actividades docentes son el entusiasmo y la pasión. Aquí hemos argüido que lo son también para la práctica de la investigación en el campo de la educación y son relevantes para cualquier actividad humana. En la actualidad, con el espíritu de las reformas educacionales y la era de la rendición de cuentas, cada vez es más difícil mantenerlos, principalmente la pasión.

En el mundo académico parece que estamos condenados al aislamiento, la rutina y la práctica de tareas que pueden estar siendo improductivas, a la muerte lenta (Fried, 1995), debido a las reglas que rigen la ciencia contemporánea, según las cuales el producto publicado parece ser la meta final de toda actividad científica y la docencia se entiende cada vez más como una actividad tecnificada.

En el primer caso nos referimos a la preeminencia de indicadores como el número de productos, el factor de impacto, el índice $h$, entre otras muestras del despropósito en el que se ha embarcado la investigación, que parecen constituir aspectos que deterioran su espíritu y propósitos 
originales, y, por supuesto, que desafían los intangibles de los que hablábamos antes. En el segundo caso aludimos al espíritu de las reformas educativas echadas a andar en los años recientes y que han simplificado la comprensión de la docencia, reduciéndola a una actividad que "puede hacer cualquiera" que cumpla los indicadores mensurables (Day, 2011; Elliot, 2000).

Además, aunque se trata de actividades que poseen el mismo origen, han seguido caminos diferentes. La investigación en educación parece cada vez más aislada y distanciada de la práctica docente, distanciamiento que, conjeturamos, obedece a la profesionalización de esta actividad científica mediante la disciplinarización, y al soslayo de sus propósitos originales.

Por disciplinarización entendemos el proceso por el que han pasado las diferentes ciencias desde que se practicaban casi como actividades artesanales, hasta constituirse en actividades profesionales. La profesionalización ha implicado la creación de instituciones, la formación de organismos, la instauración de mecanismos de comunicación, el desarrollo de programas mediante los que se reconocen, regulan e incentivan esas actividades, entre otros.

La disciplinarización ha ido reconstruyendo la torre de marfil en torno a las actividades que, aunque surgen de las prácticas, no retornan a ellas. Incluso, de alguna manera reniegan de estas asumiendo un código cada vez más complejo, centrándose en discusiones que pretenden ser cada vez más eruditas, más distanciada del lenguaje y de la vida cotidiana, aunque podrían estar siendo insustanciales para el debate académico correspondiente: aislamiento y actividades poco productivas, como mencionábamos.

Entre más disciplinarizado esté un campo científico, va alejándose más de las prácticas de las que ha surgido. Al menos en lo que concierne a la investigación realizada en el campo de la educación, esto se ha acentuado al haber asociado los productos a los criterios con los que se miden su calidad y pertinencia en la actualidad, los cuales privilegian indicadores numéricos pues representan parámetros menos complejos para realizar comparaciones internacionales (Altbach, 2015), lo cual también está en boga.

Dichos esquemas además inhiben el desarrollo de trabajos que podrían aproximar la investigación a la realidad investigada y obstaculizan la innovación, pues desde estos se promueve una atención casi completa a los productos publicados como metas finales de todo proceso científico. En razón de lo anterior, estamos ante el desafío de que nuestro trabajo se ciña 
casi exclusivamente por lo que ha sido determinado como productivismo (Miller et ál., 2011; Rego, 2014).

En el campo de la educación esto representa una verdadera paradoja pues entre más pertinente y de calidad en los términos de la ciencia actual, la investigación se distancia más de los desafíos y las prácticas que le dieron sentido. Quienes somos investigadores acatamos esas reglas, pues de ellas dependen la regulación e incentivación de lo que hacemos, aunque nos pongamos en riesgo de vaciar de significado nuestras prácticas, al menos de significado educacional. Esta es una paradoja pues entre más se va profesionalizando la investigación, más se aleja de los sistemas educativos y de las actividades educativas cotidianas.

Esta brecha es aún más amplia entre la investigación de la educación y las prácticas docentes. Aunque ambas actividades poseen intereses y propósitos comunes, incluso las razones y los significados que las sustentan parecen no hallar caminos mediante los cuales puedan reencontrarse. Si bien somos profesionales dedicados al mismo campo, y en ocasiones compartimos la formación disciplinaria y profesional, los profesores del sistema de educación básica y los investigadores que primordialmente laboramos en las instituciones de educación superior seguimos intereses y poseemos motivaciones que pocas veces coinciden.

Esto ha ocurrido a pesar de la mejora de las condiciones en las que se realiza la investigación como actividad profesional y del avance más o menos sostenido de los sistemas educativos. Por ejemplo, si nos enfocamos en la investigación, podemos constatar el aumento de investigadores, centros de investigación, programas de formación, mecanismos de comunicación y, principalmente, productos publicados. No obstante, los problemas que aquejan a la educación parecen insalvables y los principales desafíos persisten. El crecimiento casi exponencial de las publicaciones especializadas, los congresos y las jornadas parece no estar repercutiendo en la mejora de la educación - básica y superior - y se ha generado poco debate sobre este asunto (Nosei, 2013). Las publicaciones tampoco parecen estar comunicándose entre sí, ya que justamente en el campo de la educación se realizan menos réplicas a estudios y casi no se hacen metaanálisis.

Además, ni los profesores del sistema básico precisarían de la investigación para desarrollar sus actividades, ni los investigadores tendríamos como prioridad que nuestro trabajo llegue hasta el aula y las prácticas escolares, pues esto implicaría trastocar los mecanismos tradicionales de 
difusión de la investigación, que son los que le otorgan el rango de cientificidad debido a las reglas que ya señalamos, a pesar de la paradoja que representan.

La brecha y el desinterés por las actividades docentes, vistas desde las prácticas de investigación, han materializado una de las maneras de ignorar el espíritu y propósito originales de estas actividades científicas, pues, como señalamos, una buena parte de los estudios y las investigaciones del campo surgen de los desafíos cotidianos de los sistemas educativos y de las actividades docentes.

En el extremo, es hasta cierto punto desconcertante observar cómo nuestras actividades como investigadores podrían habernos sumado a la cruzada de desprestigio de la práctica docente, una especie de Friendly Fire, que es una expresión que se emplea para ironizar el hecho de no recibir apoyo de quienes presuntamente están de tu lado, o incluso recibir ataques y críticas de ellos. Aunque puede resultar incomprensible, la investigación habría concretado esa forma de desprestigio amigo, denostando las actividades docentes, sea por acción u omisión, principalmente las prácticas de los profesores del sistema básico. El ejemplo más claro lo representa el bando que habríamos tomado en la reciente crisis de la profesión magisterial, que, según algunas referencias, podría tener más medio siglo (Day, 2011; Schön, 1992).

En la cruzada del escarnio público de la docencia, desde la investigación podríamos estarnos sumando a la concepción generalizada que la desvirtúa o que, al menos, la concibe como una actividad profesional de segundo orden, apoyando con nuestra indiferencia activa o sin tomar una postura clara desde nuestro trabajo una corriente discursiva que apunta hacia su desprestigio y que señala al maestro como el único responsable de los resultados de aprendizaje.

El hecho de asumir la profesión docente como una actividad de segundo orden representa una evidencia más de la manera como habríamos ido construyendo, desde la investigación, una jerarquización de las tareas que se desarrollan en el campo de la educación. Sería una comprensión del asunto que alude a la distinción entre actividades intelectuales y artesanales, mediante la cual hacemos una diferenciación, denostando alguna de ellas. La diferenciación entre lo artesanal e intelectual puede equipararse a la distinción entre lo básico y lo aplicado en lo que concierne a la investigación. Recordemos que en los diferentes campos científicos se alega el prestigio de lo puro en demérito de lo aplicado (Becher, 2001). Basta ver la distribución de 
privilegios entre quienes son únicamente docentes y aquellos que además poseen el nombramiento de investigadores en los sistemas de educación superior.

Tal como ocurrió con la disciplinarización, en la carrera académica se va concretando algo similar a medida que avanza nuestra formación, nuestra trayectoria y nuestro relativo prestigio en el campo académico. Mientras progresamos en nuestra trayectoria, paulatinamente vamos abandonando actividades que nos apasionaban cuando aún éramos novatos, como el trabajo de campo o el laboratorio, y delegamos esas tareas a otras personas, estudiantes, asistentes de investigación, colegas más jóvenes o con credenciales menores que las propias, centrándonos en actividades más teóricas o de síntesis (Shinn, 2007).

Nosotros creemos que desde la investigación podríamos asumir enfáticamente una perspectiva en la que las tareas docentes, el trabajo del profesor de a pie, fueran concebidas como actividades superiores, como las tareas indispensables que son, como el tipo de labor inspiradora que quizás sigue siendo para los estudiantes y para los padres de familia, y para los propios maestros. Así mismo, en la carrera productivista hemos dejado tal vez de creer que la práctica docente, las actividades que se llevan a cabo en cualquiera de los niveles y modalidades educativas, fortalece nuestra formación continua como investigadores y puede dotar de significado nuestras prácticas de significado educacional.

La vuelta a la práctica docente como herramienta de formación continua puede ser una manera de encontrar el contrasentido a la creciente rutinización y burocratización de la investigación, al abandono de sus propósitos más genuinos, y al aislamiento de las prácticas que le dieron origen y sentido a una buena parte de los trabajos de investigación que realizamos. El regreso constituiría una forma de revitalizarse como investigador pues, como decíamos al inicio, algunos de los intangibles que definen y renuevan nuestra actividad profesional son el entusiasmo y la pasión.

Queremos desandar el camino que nos ha llevado a la búsqueda del producto por el producto mismo para emprender otro rumbo que quizás nos acerque a los propósitos iniciales de la investigación en nuestro campo, que nos permita enriquecer la noción de la educación como una praxis que pretende generar oportunidades de aprender y crear redes colaborativas con otros actores de la educación, como señala Nosei (2013). Es importante asumir que la práctica docente puede ser generadora de reflexiones que complementen nuestra formación y consoliden la investigación como fundamento de la docencia, y viceversa. 


\section{Cierre o agenda de discusión}

La estrategia viceversa la propusimos en el sentido de pensar que la formación continua de los profesores se puede configurar en un espacio-tiempo de investigación de la propia práctica pedagógica, y que la investigación como forma de problematización de la cotidianeidad docente posibilita la misma investigación. Esto lo hacemos a pesar de que en el campo de la educación a la profesión docente no se le concede la misma importancia que a las actividades científicas, aunque se trate de tareas complementarias que dependen una de la otra y se fundamentan mutuamente.

Un aspecto relevante para que pueda existir esta relación, una posibilidad para la dinámica viceversa que hemos propuesto, es la constitución del profesor como un sujeto reflexivo. A partir de las ideas de Schön (1992, 2000) y Zeichner (1992, 1993, 2008), subrayamos que reflexionar no se reduce al simple acto de pensar, es aún más profundo que eso. La reflexión puede ser entendida como un fenómeno humano que lleva a percibir, examinar, observar, ponderar, buscar entender las situaciones que generan la necesidad de reflexionar. Como mencionábamos, la reflexión es el análisis constante de los acontecimientos y el autoanálisis que ocurre en el proceso, al igual que sus efectos en la vida cotidiana. Constituirse como profesor reflexivo es asumir una postura activa, dialógica y transformadora.

La relación docencia/formación continua mediante el proceso reflexivo intenta superar la racionalidad técnica que en su origen se centra en la "instrumentalización”, lo que artificializa el proceso de enseñanza y aprendizaje, llevándolo fuera del contexto en el que se generó. Entendemos que esta práctica está muy presente en las escuelas y que, en términos generales, se desarrolla en las instituciones educativas, pero esto no nos extraña, pues es la manera como los maestros fueron y podrían todavía estar siendo formados. Esto representa un desafío para la formación del profesorado en la época contemporánea.

El mito de Sísifo como metáfora nos llevó a pensar de modos diferentes los engranajes que sustentan los procesos de docencia, formación continua, investigación y viceversa, ya sea aludiendo a la persistencia o admitiendo la insensatez que subyace a las prácticas en cuestión. Ambos, persistencia e insensatez, constituyen la trama de las relaciones del "objeto" aquí problematizado. 
Empleamos el mito como epígrafe debido a la manera como se originó la colaboración entre nosotros como autores. Comenzamos a pensar el texto compartiendo nuestros intereses de investigación, formación continuada en el caso de Andrade, uso de la investigación en el caso de Moreles. Nuestra lengua nativa es diferente, aunque, al igual que las realidades educativas latinoamericanas, portugués y español son muy semejantes. Además, en las diferencias existen aspectos significativos que aquí es preciso notar, pues nos llevaron a interpretaciones un poco distintas del mito y del mismo texto que hemos escrito. Andrade optó por la perseverancia de rodar la piedra cada vez que cae cuando Sísifo casi la lleva a la cumbre; Moreles asumió esta cuestión como una necedad, como una metáfora de las actividades que no concretan las metas que inicialmente se plantean quienes las realizan. Como esperamos haya ocurrido con el texto, al final ambas interpretaciones son complementarias.

Nuestras interpretaciones del mito de Sísifo son diferentes porque en la superficie de lo que se piensa y se escribe hay un encuentro con la diferencia - construcciones subjetivas intransferibles - que lleva a percibir el mundo de un modo único y, justamente por eso, bello. Es en este entre-espacio que se encuentra la posibilidad de la percepción de la estética de lo cotidiano, desde la cual argumentamos aquí, y que puede ser un lugar de cuestionamiento y de atención a lo que acontece cotidianamente en el espacio del aula y en el contexto de las instituciones educativas.

De hecho, habría posibilidades formativas si los sujetos fueran afectados de alguna manera por lo cotidiano, y, en virtud de eso, transformados para percibir otros modos de mirar la profesión y, por qué no, la vida. Retomamos la idea planteada en la introducción con la figura 1, aunque ahora incorporando dos elementos que permean el asunto que hemos problematizado en el presente escrito.

\section{INSERTAR FIGURA 2}

Figura 2. Representación global

Fuente: elaboración propia

Profesión y vida son dos instancias que conforman nuestra existencia y están siempre en tensión. La cuestión es si, como en el mito de Sísifo, rodaremos la piedra y, además de eso, seremos 
capaces de identificar la persistencia o insensatez en este acto. Con la figura 2 pretendemos invitar a pensar que este movimiento viceversa depende de una serie de situaciones que conforman la red de relaciones que acontecen en nuestras escuelas. Algunas tensiones son de orden personal, ligadas a la vida, otras son del orden institucional, político, económico e incluso de comportamiento, y por lo tanto están relacionadas con la profesión. Hay características específicas en cada uno de los instancias, sin embargo, ninguna de ellas actúa de manera aislada, están siempre en relación, lo cual tratamos de ilustrar con las figuras 1 y 2, sus distanciamientos, complementariedades y viceversa.

\section{Referencias}

Altbach, P. G. (2015). What counts for academic productivity in research universities? International Higher Education, (79), 6-7. https://doi.org/10.6017/ihe.2015.79.5837

Becher, T. (2001). Tribus y territorios académicos. La indagación intelectual y las culturas de las disciplinas. Gedisa.

Bourdieu, P. (2004). Os usos sociais da ciência: por uma sociologia clínica do campo científico. UNESP.

Day, C. (2011). Pasión por enseñar: la identidad personal y profesional del docente y sus valores. Narcea.

Elliot, J. (2000). El cambio educativo desde la investigación-acción (3 $3^{\mathrm{a}} \mathrm{ed}$.). Morata.

Estrela, M. T. y Estrela, A. (2006). A formação contínua de professores numa encruzilhada. En R. Bizarro y F. B. Silva, Formação de professores de línguas estrangeiras: reflexões, estudos e experiências. Porto Editora.

Ferreira, A. B. H. (2010). Mini Aurélio: o dicionário da língua portuguesa (8 a ed.). Positivo.

Freire, P. (1990). La naturaleza política de la educación. Cultura, poder y liberación. Paidós.

Fried, R. L. (1995). The passionate teacher. Beacon Press.

Marques, M. O. (2011). Escrever é preciso: o princípio da pesquisa. Vozes.

Miller, A. N., Taylor, S. G. y Bedain, A. G. (2011). Publish or perish: academic life as management faculty live it. Career Development International, 16(5), 422-445. https://doi.org/10.1108 / 13620431111167751 
Nosei, C. (2013). Pensando la tarea de investigar. Praxis educativa, (17), 51-57. http://170.210.120.129/index.php/praxis/article/download/580/549

Pacheco, J. E. (2009). Tarde o temprano (Poemas 1958-2009). Fondo de Cultura Económica.

Pereira, E. A. T. (2015). O conceito de campo de Pierre Bourdieu: possibilidade de análise para pesquisas em história da educação brasileira. Revista Linhas, 16(32), 337-356. https://doi.org/10.5965/1984723816322015337

Pereira, M. V. (1996). A estética da professoralidade. Um estudo interdisciplinar sobre a subjetividade do professor [Tesis de doctorado]. Pontifícia Universidade Católica.

Rego, T. C. (2014). Produtivismo, pesquisa e comunicação científica: entre o veneno e o remédio. Educação e Pesquisa, 40(2), 325-346. https://doi.org/10.1590/S151797022014061843

Richter, I. M. (2003). Interculturalidade e estética do cotidiano no ensino das artes. Mercado das Letras.

Schön, D. (1992). La formación de profesionales reflexivos. Hacia un nuevo diseño de la enseñanza y el aprendizaje de las profesiones. Paidós.

Schön, D. (2000). Educando o profissional reflexivo: um novo design para o ensino e a aprendizagem. Artes Médicas Sul.

Shinn, T. (2007). Jerarquías de investigadores y formas de investigación. Redes, 12(25), 119-63. https://www.redalyc.org/pdf/907/90702505.pdf

Stenhouse, L. (2007). La investigación como base de la enseñanza (6 $6^{\mathrm{a}} \mathrm{ed}$.). Morata.

Zeichner, K. M. (1992). Novos caminhos para o practicum: uma perspectiva para os anos 90. En A. Nóvoa, Os professores e sua formação. Educa.

Zeichner, K. M. (1993). A formação reflexiva de professores: ideias e práticas. Educa.

Zeichner, K. M. (2008). Uma análise crítica sobre a "reflexão" como conceito estruturante na formação docente. Revista Educação e Sociedade, 29(103), 535-554. http://www.cedes.unicamp.br

\section{Notas}

i Significado etimológico, https://www.significados.com.br/pesquisa

ii Significado etimológico, http://origemdapalavra.com.br/site/palavras/pesquisar/ 